\title{
An Efficient Energy Aware Link Stable Multipath Routing Protocol for Mobile ad hoc Networks in Urban Areas
}

\author{
Salah Eddine Benatia, Omar Smail, Boudjelal Meftah, Mohammed Rebbah, and Bernard Cousin
}

\begin{abstract}
Ad Hoc wireless mobile networks are characterized by a lack of central administration and the fact that any element of the network, being very mobile, is susceptible to disappear. In an Ad Hoc network, all the elements must cooperate in order to establish a temporary network to communicate. This communication is affected by the links stability mainly in a restricted environment such as a city and the depletion of the batteries energy of the nodes. These factors degrade the ad hoc networks performance.

We propose in this paper a stable routing protocol with weak energy consumption for urban areas in order to improve these performances. The proposed solution is a multipath protocol named ESMRua (Efficient energy aware and Link Stable Multipath Routing Protocol in urban areas). ESMRua uses a path selection scheme based on energy constraint, signal quality and link stability. For the link stability, three calculation variants are used. ESMRua will be designed for realistic mobility, contrary to the main existing protocols which are designed to the random mobility models; these models have unrealistic behaviors such as a brusque acceleration and a sudden stop. Simulation results show that our multipath protocol enhances the performance of the ad hoc networks, mainly in terms of routing overhead, energy consumption and network reliability.
\end{abstract}

Keywords - Mobile ad hoc network, Multipath routing, Mobility model for urban areas, Energy efficiency, Link stability and quality, Network reliability.

\section{INTRODUCTION}

$\mathrm{W}$ IRELESS technology offers great flexibility of use and networking that allows users to access information regardless of time factors and location. This technology continues to grow, by providing several advantages to humanity in different areas to save several constraints such as hardware, the duration of networking and uninstallation of equipment, as well as great flexibility in terms of communication between nodes. It offers also adaptation mechanisms to the various changes caused by the mobility of mobile elements within the network. Among the mobile networks, we have Ad Hoc networks. An Ad Hoc network

Paper received December 10, 2019; revised March 18, 2020; accepted April 02, 2020. Date of publication July 31, 2020. The associate editor coordinating the review of this manuscript and approving it for publication was Prof. Grozdan Petrović.

Salah Eddine Benatia, Omar Smail, Boudjelal Meftah and Mohammed Rebbah are with Faculty of exact sciences. Computer Science Department. University Mustapha Stambouli, Mascara, Algeria.

(e-mail: se.benatia@univ-mascara.dz, o.smail@univ-mascara.dz, boudjelal.meftah@univ-mascara.dz,rebbahmed@univ-mascara.dz).

Bernard Cousin is with IRISA/University of Rennes 1, France (e-mail: b.cousin@irisa.fr). is a set of mobile entities interconnected by a wireless technology forming a temporary network without the aid of any administration or any fixed support.

In an Ad Hoc network, all nodes must cooperate in order to create a temporary architecture to communicate effectively. To create this architecture in order to route the data, Ad Hoc networks must use an efficient routing protocol. Several routing protocols for ad hoc networks have been developed [1-3]. These protocols can be reactive, proactive or hybrid [4]. However, among the limitations that are reproached to these protocols, are the lack of support for the problem of energy consumption and the mobility of the nodes during data transfer. While some nodes have little involvement in routing, others are heavily congested and routing most of the network traffic. Due to this inhomogeneous charge distribution, charged nodes consume their limited hardware resources quickly and lead to heavy congestion.

All nodes in an Ad Hoc network can be dynamically connected. It is usually possible to establish more than one path between a source and a destination. When this property of Ad Hoc networks is used in the routing process then we evoke the multipath routing. The purpose of the multipath technique is to find alternative paths through a single path discovery process in order to find better adaptation to frequent topology changes, link breaks, and network overhead. This technique also attempts to improve the quality of service by reducing the end-to-end delay during data transfer. In order to improve the performance of ad hoc mobile wireless networks, we propose a multipath, stable and low-energy consumption routing protocol. Our Energy aware and Stable Multipath Routing Protocol (ESMRua) routing protocol use a selection strategy based on energy constraints and link stability. ESMRua is an extension of our work [5], this extension mainly concerns integration in paths selection of the signal quality and link stability, calculated with three variants based on standard deviation, average absolute deviation and exponentially weighted moving deviation.

In order to design or thoroughly study a new protocol for networks, it is important to simulate this protocol and evaluate its performances. The protocol simulation has several key parameters, among these parameters the mobility model. The mobility model is designed to describe the movement pattern of mobile nodes, their location, speed and acceleration changing over time. Since mobility models have an important role in determining the performance of the protocol, it is desirable that mobility models simulate 
the mobility in real-life applications reasonably. Otherwise, observations made and conclusions drawn from simulation studies can be misleading. The mobility model frequently used in Ad Hoc network simulations is the Waypoint random model [6], in which nodes move independently to a random destination with a randomly chosen speed. The simplicity of the Waypoint model is perhaps the reason for its widespread use in simulations. However, Ad Hoc networks can be used in urban areas where mobility models are complex.

Our ESMRua protocol will be simulated on the basis of a realistic mobility model, namely the Manhattan model [6], designed for urban cities, unlike most protocols in the literature that are based on random mobility models, which adopt unrealistic behaviours such as abrupt stopping, sudden acceleration and sudden turning. Through the evaluation, we notice that our ESMRua protocol improves the network performance in terms of energy, successfully received packets and control packets.

The article is organized as follows. Section 2 presents a state of the art regarding the multipath routing protocols stable and energy efficient for mobile ad hoc networks. Section 3 details the design of our ESMRua protocol. Section 4 provides the evaluation of the performance of the proposed protocol, finally section 5 concludes this paper.

\section{MULTIPATH ENERGY-AWARE AND STABLE ROUTING PROTOCOLS}

The routing protocols have an important part in node energy conservation during the ad hoc communication network. Routing techniques can extend the nodes lifetime as well as the entire network.

Many researches have studied routing based on the energy conservation [7]. These protocols can use for routing: a single path or multipath. A multipath routing can be an effective way to save energy in ad hoc networks.

Several multipath protocols [8-10], that conserve the networks energy, have been proposed in order to extend network lifetime by minimizing link breaks. All of these works solve the energy conservation problem, but the majority of the techniques used are based on the remaining energy only; they are not designed to discover the best path between source and destination nodes. On one hand, when a node accepts routing requests because it has enough residual battery capacity, routing traffic is routed through this node. On the other hand, many energy saving mechanisms/algorithms neglect the power consumption induced by each node own message sending, which may cause network partitioning due to node battery depletion. Indeed, it decreases network performance. Hence, balanced energy consumption is a remedy for these natures of problems. Finally, another problem of these routing protocols schemes is that they do not consider the paths stability in their path establishment process. The nodes mobility makes the network topology very unstable and causes path-breaking. Several protocols [11-13] have been proposed to contribute to solving this problem. In most of these routing schemes, a new path-discovery process is launched once a path rupture is detected. This increases delay and causes node resources wastage which may reduce network lifetime. The multipath routing is an effective concept in mobile ad hoc networks to adjust the problem of frequent topology changes in the network caused mainly by link failures. Many existing multi-path routing protocols [14-16], are primarily related to link instability issues. However, these protocols do not take into consideration the energy consumption, implying an overload due to the number of control messages sent when nodes disconnecting due to their battery depletion. Other weakness of these techniques, they have been compared only with protocols which do not consider the link stability and the multipath principle; therefore these routing protocols performance evaluations are not fair. A few multipath protocols using the link stability and the energy consumption as criteria to select the best paths, for routing data packets have been proposed; we cite [17-18]. The majority of these protocols were simulated in unrealistic environments with not practical nodes behavior, such as sudden stop and change of direction under high speed. We will use EESMR [17] as a reference for our protocol routing performance evaluation because it aims to purpose the performance of the ad hoc network and have the same characteristics as our solution, namely its multipath concept; reactivity and it uses two selection criteria: link stability and node battery energy.

\section{EFFICIENT ENERGY-AWARE AND LINK STABLE} Multipath Routing Protocol In URban AREAS

The aim of this article is to develop an efficient routing protocol, named Efficient energy-aware and link Stable Multipath Routing protocol adapted to urban areas (ESMRua), for ad hoc networks. ESMRua is a reactive (on demand) and multipath routing protocol that selects the energy-efficient path with stable links. It is designed to operate in urban areas.

\section{A. Multipath routing discovery}

Our path discovery process is based on reactive routing protocols. When the source node requires a path toward the destination node, the source consults its routing table for any accessible path toward to the destination node. If there is no path or it is invalid, the source node launches the path discovery: it diffuses a message of Route Request (RREQ) to its neighbor's nodes. When a node gets a RREQ message, it guarantees that the RREQ received is not a copy of the RREQ by examining the source identifiers of the RREQ, in order to anticipate looping routes. When the nodes receive the RREQ message, they retransmit to its neighbor nodes to discover the path toward the destination node. Once the destination node gets the first RREQ message, it waits for a specific time to collect RREQs messages and responds by sending Route Reply (RREP) messages. Our solution utilizes the destination node sequence number as [8], to get the fresh paths, which avoids loops. We use also, an advertised hop count to deal with the various routes for a similar RREQ identifier and the same sequence number. The advertised hop count has as value the longest path hop count. The advertised hop count is reset when the sequence number changes and does not change for this sequence number. In fact, an alternate node creates an entry in its routing table for a neighbor node only if the neighbor node has a lower announced hop count. The discovery of the 
multipath must consider the sequence number to guarantee the maximum value of the hop count and the freshness of paths, signified by advertised_hopcount and seqnum.

\section{B. Multipath routing selection}

This section represents a multipath selection procedure. When the source node receives the first RREP message (Route Reply), it waits an amount of time (RREP_Wait_Time) to receive more RREPS (Route Request) before path selection. The best path selection between a source node $\mathrm{s}$ and destination node $\mathrm{d}$ is based on energy consumption and path stability. Two functions are defined: the cost function $f e p_{j}(t)$ which represents the energy path $j$ at time $t$ and the cost function $f s p_{j}(t)$ which represents the stability of path $j$ at time $t$.

\section{B.1 Energy-aware cost function}

Let $f e p_{j}(t)$, the minimum nodes energy costs constituting the path $j$ for a source $s$ to destination $d$ at time $t$, be expressed as:

$$
f e p_{j}(t)=\min _{i=1}^{n-1}\left(\operatorname{fen}_{i, j}(t)\right)
$$

where $f e n_{i, j}(t)$ represents the energy cost function of node $i$ forming the path $j$, formally:

$$
\operatorname{fen}_{i, j}(t)=\frac{\operatorname{Elev}_{i, j}(t)}{D R_{i, j}(t)} .
$$

$\operatorname{Elev}_{i, j}(t)$ denotes the node $i$ energy level belonging to the path $j$ at time $t$, given by:

$$
\operatorname{Elev}_{i, j}(t)=\frac{E_{i, j}(t)}{E_{\text {average }}}
$$

where $E_{i, j}(t)$ represents the residual energy of the node $i$ belonging to the path $\mathrm{j}$ at time $\mathrm{t}$ and $E_{\text {average }}$ is the nodes average residual energy of nodes that participated in the multipath discovery process between the source node $s$ and the destination node $d . D R_{i, j}(t)$ is the drain rate of the node $i$ forming the path $j$ at time $t$, defined as the rate at which energy is consumed by a given node, mainly when a node is used by different paths than source $s$ and destination $d$.

\section{B.2 Link stability aware cost function}

To evaluate the stability of links in mobile ad hoc networks, we take into consideration the nodes mobility as the main metric. Our protocol mainly utilizes message delay between the sending and receiving time to measure the link stability. We exploit the messages of the mutipath discovery and hello messages for collecting position information of the neighbors nodes, to avoid the overhead which will be generated by specific messages.

\begin{tabular}{|l|l|l|l|l|}
\hline Destination & $\begin{array}{l}\text { Destination } \\
\text { sequence } \\
\text { number }\end{array}$ & $\begin{array}{l}\text { Hop } \\
\text { count }\end{array}$ & Distance $_{\mathrm{i}, \mathrm{j}}$ & $\begin{array}{l}\text { Expiration } \\
\text { timeout }\end{array}$ \\
\hline
\end{tabular}

Fig. 1. A node structure entry of a HELLO packet.

Each node adds its node destination, hop count and sequence number into the original hello message. In order to collect distances that separate nodes, we added a new field to the Hello message named Distance $_{i j}$, noted by $d_{i j}$, see Fig. 1.

Fig. 2 shows the structure of node $i$ routing table. There is an entry for each destination node attainable by the node $i$. The field Route_list contains the neighbor nodes of node $i$ leading to that destination. Each neighbor node of this list, is identified by its nexthop address, and the hopcount field defined by the number of hops to achieve that destination through this neighbor. We propose to add two new fields to the basic routing table. The first new field $E_{j}$ defines the residual energy of a neighbor node and the second new field distance_list denotes the registered distances over different time between the node $i$ and its neighbors.

\begin{tabular}{|l|}
\hline Destination \\
\hline Sequence_number \\
\hline Advertised hopcount \\
\hline Route_list \\
$\left\{\left(\right.\right.$ nexthop1, hopcount $1, \mathrm{E}_{1}$, \\
distance_list $\left.\left\{\left(\mathrm{d}_{\mathrm{i} 1}, \mathrm{t}_{1}\right),\left(\mathrm{d}_{11}, \mathrm{t}_{2}\right), \ldots\right\}\right)$, \\
(nexthop2, hopcount $2, \mathrm{E}_{2}$, \\
distance_list $\left.\left.\left\{\left(\mathrm{d}_{\mathrm{i} 2}, \mathrm{t}_{1}\right),\left(\mathrm{d}_{\mathrm{i} 2}, \mathrm{t}_{2}\right), \ldots\right\}\right), \ldots\right\}$ \\
\hline Expiration timeout \\
\hline
\end{tabular}

Fig. 2. Structure of a routing table entry for ESMRua.

The cost function stability of path $j$ at time $t$, denoted by $f_{s p_{j}}(t)$ is the maximum of the links stability costs constituting the path $j$, given by:

$$
f_{s p_{j}}(t)=\max _{i=1}^{n-1}\left(f_{s} l_{i, j}(t)\right)
$$

To calculate this link stability cost, we assume two nodes $i$ and $j$. The node i sends periodically a control packet to the node $j$, when the node $j$ receives this packet, it computes its coordinates then sends it back to the node $i$. Using these coordinates, the node $i$ computes the distance that separates it from the node $j$ noted by $d_{i, j} . f_{s} l_{i, j}(t)$ represents the link $i, j$ stability cost function at time $t$, given by:

$$
f_{S} l_{i, j}(t)=\frac{S D l_{i, j}(t)}{M l_{i, j}(t)} S_{q}
$$

The cost function $f_{S} l_{i, j}(t)$ represents the relative standard deviation, also known as a coefficient of variation. It is a standardized measure of dispersion of a probability distribution. The coefficient of variation formulated by $f_{S} l_{i, j}(t)$ is used to quantify the measurement accuracy. For our protocol proposition, it is the distance measurement between two neighbor nodes. If the $f_{s} l_{i, j}(t)$ tends to 0 , this trend indicates a good distribution of distances, showing that the link is stable. But if it tends to 1 or to infinity, this indicates a poor distribution, showing the link is unstable. Finally, we introduce the factor $S_{q}$, the indicator of the signal quality. This distinguishes the signals according to their assured quality in terms of throughput, transmission delay, etc.

We propose three variants to calculate the coefficient of variation, based on standard deviation, Average absolute deviation, and exponentially weighted moving deviation.

\section{B.2.1 The coefficient of variation, based on standard deviation}

For this variant, $M l_{i j} j(t)$ (see equation 5) indicates the $n$ recorded distances mean between the node $i$ and node $j$, formulated as:

$$
M l_{i, j}(t)=\frac{\sum_{t=t 1}^{t n} d_{i, j}(t)}{n}
$$

$S D l_{i, j}(t)$ defines the recorded distances standard deviation 
between the node $i$ and node $j$, given by:

$$
S D l_{i, j}(t)=\sqrt{V l_{i, j}(t)}
$$

and $V l_{i, j}(t)$ represents the variance of the distances $d_{i, j}(t)$, defined as:

$$
V l_{i, j}(t)=\frac{1}{n} \sum_{t=t 1}^{t n}\left(d_{i, j}(t)-M l_{i, j}(t)\right)^{2}
$$

The standard deviation is the most used data dispersion measurement parameter. Its meaning is however far from obvious. It is fully justified in the case where the distribution of the values of the observed distribution is Gaussian or, at least, symmetrical and unimodal. In these cases, he can define confidence intervals around the mean.

\section{B.2.2 The coefficient of variation, based on average absolute deviation}

In this second case, the mean of the $\mathrm{n}$ distances recorded between the node $i$ and node $j$ is calculated in the same way as the previous section (B.2.1).

$S D l_{i, j}(t)$ denoted the average absolute deviation of the distances recorded between the node $i$ and node $j, S D l_{i, j}(t)$ is given by:

$$
S D l_{i, j}(t)=V l_{i, j}(t)=\frac{1}{n} \sum_{t=t 1}^{t n}\left|d_{i, j}(t)-M l_{i, j}(t)\right|
$$

The interest of the average absolute deviation is to be easy to compute and simple to interpret any data distribution. Unlike the standard deviation which is much more used although its interpretation is much less obvious.

\section{B.2.3 The coefficient of variation, based on exponentially weighted moving deviation}

For the third and last, $M l_{i, j}(t)$ (see equation 5) represents the exponential moving average of the $n$ distances recorded between the node $i$ and node $j$, formulated as follows:

$$
M l_{i, j}(t)= \begin{cases}d_{i, j}(1), & t=1 \\ \sigma d_{i, j}(t)+(1-\sigma) M l_{i j}(t-1), & t>1\end{cases}
$$

$\sigma$ is between 0 and 1 and depends on the number of the collected distances. $S D l_{i, j}(t)$ has the same notation as presented in section (B.2.1), it represents the exponentially weighted moving deviation variance of the recorded distances between the node $i$ and node $j$. And $V l_{i, j}(t)$ represents the exponentially weighted moving variance of the distances $d_{i, j}(t)$, defined as:

$$
\begin{gathered}
V l_{i, j}(t)=(1-\sigma)\left(V l_{i, j}(t-1)+\sigma \delta^{2}\right) \\
\delta=d_{i, j}(t)-M l_{i, j}(t-1)
\end{gathered}
$$

with $M l_{i, j}(1)=d_{i, j}(1)$ and $V l_{i, j}(1)=0$.

This variance is called moving variance because it is recalculated continuously, using each calculation a subset of elements in which a new element replaces the oldest or adds to the subset. This type of variance is generally used as a method of measuring time series.

\section{B.3 Objective problem formulation}

The multipath selection of our protocol is based on the nodes energy consumption and the link stability of their path links. The corresponding objective function $f p_{j}(t)$ of path $j$ at time $t$ is defined by combining the energy cost function defined in formula (1) and the path cost function stability formulated by formula (4):

$$
f p_{j}(t)=\alpha f e p_{j}(t)+\frac{\beta}{f s p_{j}(t)}
$$

Our multipath selection idea is based on sorting all discovered paths between a source node $s$ and a destination node $d$ by the descending value of $f p_{j}(t)$. The path with the maximum value of $f p_{j}(t)$ is chosen to forward the data packets. At last, we note that the weights $\alpha$ and $\beta$ are chosen satisfying the condition $\alpha+\beta=1$.

\section{Multipath routing maintenance}

Route error detection in ESMRua is like route error detection in [8]. It is launched when a link breaks between two nodes in a current path. When a node neighbor does not react to three progressive HELLO messages sent by a node in an active path, the link is considered broken. In this case, it erases the active path from its routing table and sends a Route ERROR message (RERR) to the source node.

\section{Performance Evaluation of ESMRua}

This section presents the performance evaluation of our ESMRua protocol to demonstrate the effectiveness of the proposed solution. First, we present and define the performance evaluation metrics used, and then we evaluate our protocol with its three variants by comparing it with a protocol using the same routing principle EESMR [17], applying two models of the mobility: Random Waypoint and Manhattan Mobility [6]. The evaluation is accompanied by a detailed analysis and a discussion of the obtained results.

\section{A. Mobility model}

The routing protocol performance designed for the ad hoc networks can change significantly when it is tested with different mobility models, but also when the same model of the mobility is used with various parameters. The performance should be evaluated with the model of mobility which is near to the real scenario; this can facilitate a fair evaluation of the ad hoc network routing protocol. The Grid Manhattan model [6], it's also named the Urban Area Model. It consists of a number of horizontal and vertical streets forming a grid. Each mobile node can move along the grid of vertical and horizontal roads (streets). At the intersection of the vertical and horizontal street, the mobile node can turn right, left or straight by a probabilistic approach. The model Manhattan grid mobility creates a realistic movement for a part of a city since it limits the mobile node displacements. These mobile nodes do not move freely without worrying about the streets of the city, obstacles and traffic regulations.

\section{B. Performance parameters}

We evaluate three performance metrics. The energy consumption represents the average of the consumed energy by the participating nodes in the transfer packets (control and data) to the destination node. The packet delivery ratio (PDR) is the ratio of data packets received by the destination node to those produced by the source node. Finally the normalized routing overhead (NRO) is computed by dividing the number of routing packets sent by the data packets number received during the simulation. 


\section{Performance evaluation}

The simulations concern three variants of our protocol: ESMRua-SD (standard deviation), ESMRua-AAD (average absolute deviation) and ESMRua-EWMD (exponentially weighted moving deviation). The values of simulation parameters are summarized in Table 1.

TABLE 1: Simulation PARAMETERS.

\begin{tabular}{|c|c|c|}
\hline $\begin{array}{l}\text { Mobility } \\
\text { model }\end{array}$ & Parameter & Value \\
\hline \multirow{11}{*}{$\begin{array}{c}\text { Random } \\
\text { Waypoint } \\
\text { And } \\
\text { Grid } \\
\text { Manhattan }\end{array}$} & Channel type & Wireless \\
\hline & Routing protocol & EESMR/ESMRua \\
\hline & Simulation time & $250 \mathrm{~s}$ \\
\hline & Nodes number & $100-200$ \\
\hline & Radio propagation model & $\begin{array}{r}\text { Propagation/Two } \\
\text { Ray Ground }\end{array}$ \\
\hline & Max packet In ifq & 100 \\
\hline & Terrain range & $1000 \mathrm{~m} \times 1000 \mathrm{~m}$ \\
\hline & Transmission range & $250 \mathrm{~m}$ \\
\hline & RREP Wait Time & $0.5 \mathrm{~s}$ \\
\hline & $\alpha, \beta$ & $0.5,0.5$ \\
\hline & $S_{q}$ & $0<S_{q}<1$ \\
\hline \multirow{9}{*}{$\begin{array}{c}\text { Grid } \\
\text { Manhattan }\end{array}$} & Circular & false \\
\hline & $\begin{array}{l}\text { Dimension of movement } \\
\text { output }\end{array}$ & $2 \mathrm{D}$ \\
\hline & Xblocks, Yblocks & 11,7 \\
\hline & UpdateDist & 5.0 \\
\hline & TurnProb & 0.5 \\
\hline & SpeedChangeProb & 0.2 \\
\hline & AvgSpeed & $5,10,15,20$ \\
\hline & PauseProb & 0.05 \\
\hline & MaxPause & 1 \\
\hline
\end{tabular}

(a) Number of network nodes: 100 nodes

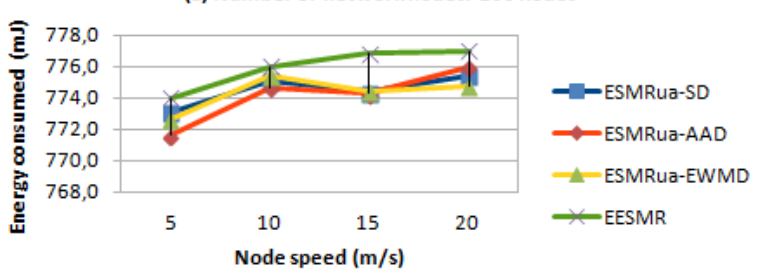

(b) Number of network nodes: 200 nodes

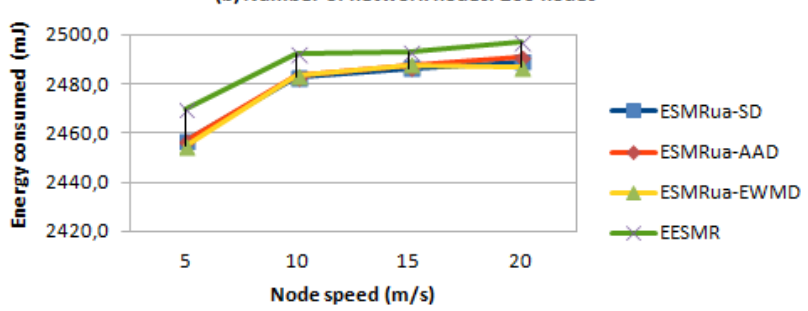

Fig. 3. Energy consumed versus node speed (based on the Manhattan model).

To evaluate ESMRua, we use ns-2 [19], the network simulator. The model Random Waypoint and Manhattan mobility model are used. The shared radio model used is similar to the IEEE 802.11 standard. The number of simulation runs is 10 per value, and the source node and destination node are randomly chosen. It is assumed that a receiver consumes $910 \mathrm{~mW}$ and for transmission $1300 \mathrm{~mW}$ [20]. We introduced the weighting values ( $\alpha$ and $\beta$ ) equal to 0.5 , so as not to favor the node stability or energy; In order to respect the basic principle of the EESMR protocol, which also does not promote the node stability or energy.

Fig. 3 shows the energy consumed by ESMRua with the three variants of calculating the coefficient of variation and EESMR protocols using the Manhattan model. ESMRua has a limited performance when the nodes have a low moving speed or when there is a small number of network nodes, but as the number of nodes increases and as the speed increases (see Fig. $3(a, b)$ ), links become unstable, path becomes longer. Mobile nodes consume more energy, which favour failures of links this highlights our solution. We can see that the energy consumed in ESMRua is less than that consumed by EESMR, this prolongs the lifetime of individual node and hence the entire network lifetime.

The PDR (Packet delivery ratio) is shown in Fig. 4 with different number of network nodes for various moving speeds. The PDR of ESMRua for three variants of calculating the coefficient of variation is higher than EESMR for the Manhattan model. Our protocol successfully receives more packets compared to EESMR protocol mainly for a medium or high number of network nodes, which ensures a good reliability of our protocol for not-random models of mobility designed for cities. ESMRua selects the best paths in terms of energy, stability and signal quality.

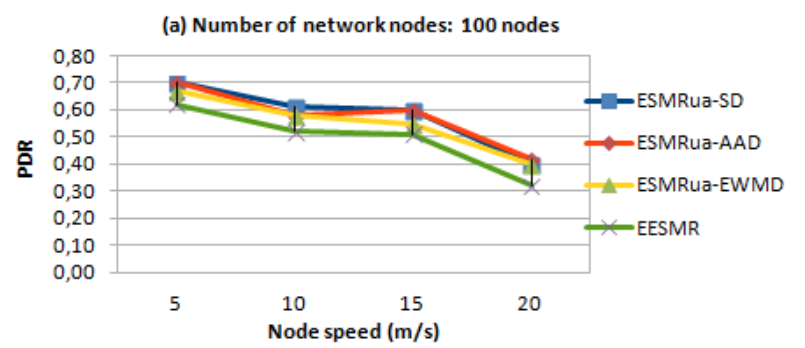

(b) Number of network nodes: 200 nodes

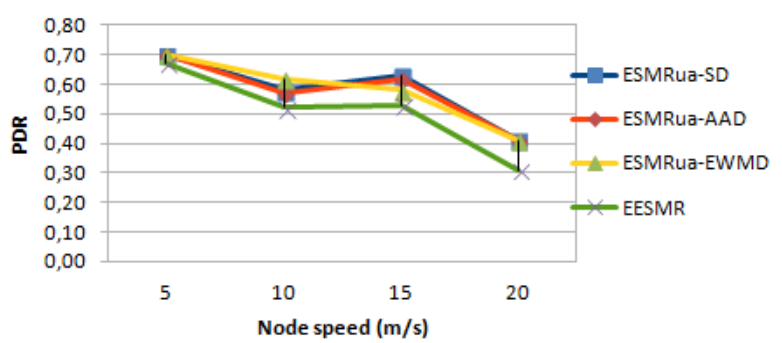

Fig. 4. PDR versus node speed (for the Manhattan model).

Fig. 5 shows NRO (Normalized Routing Overhead). In all cases with different number of network nodes for various moving speeds based on the Manhattan model, ESMRua demonstrates significantly lower NRO than EESMR (with a slight performance to the average absolute deviation variant). NRO of EESMR is much higher than ESMRua protocol, so due to the high links breaks caused by a city restriction, EESMR requires more routing packets to maintain transmission of data packets. Our protocol ESMRua is better suited to routing in a restricted city, by selecting the most stable paths with a better signal quality.

Fig. 6 and Fig. 7 show the different simulations of our protocol with the three variants compared with the EESMR protocol based on a Random Waypoint model. The results of the energy consumed and the PDR, show that our protocol with these three variants as the EESMR protocol, provides the same performance with a slight difference. This indicates that in an environment without restrictions, the EESMR protocol is also efficient as ESMRua is, but for non-random mobility in urban areas, the previous results show that our ESMRua protocol is more efficient. 


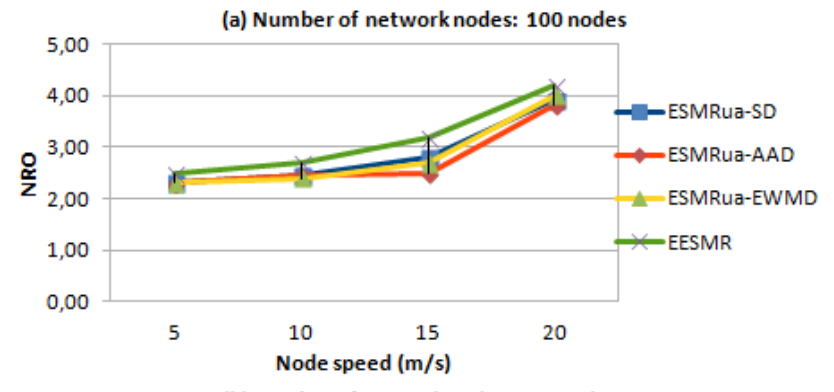

(b) Number of network nodes: 200 nodes

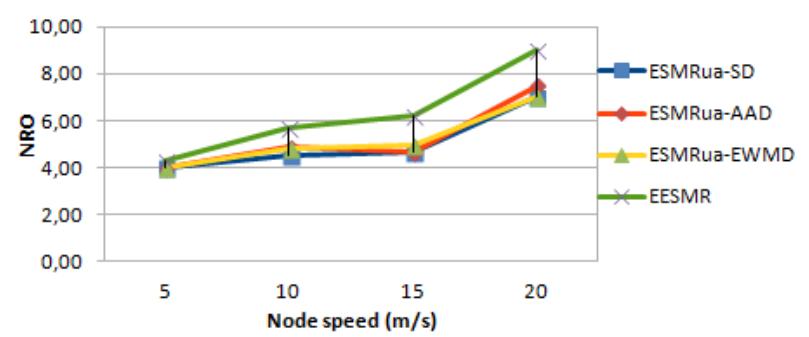

Fig. 5. NRO versus node speed (for the Manhattan model).

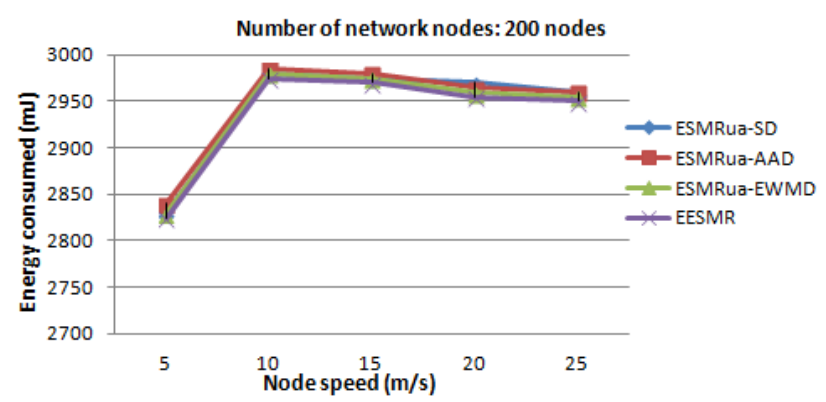

Fig. 6. Energy consumed versus node speed (based on a RWP model).

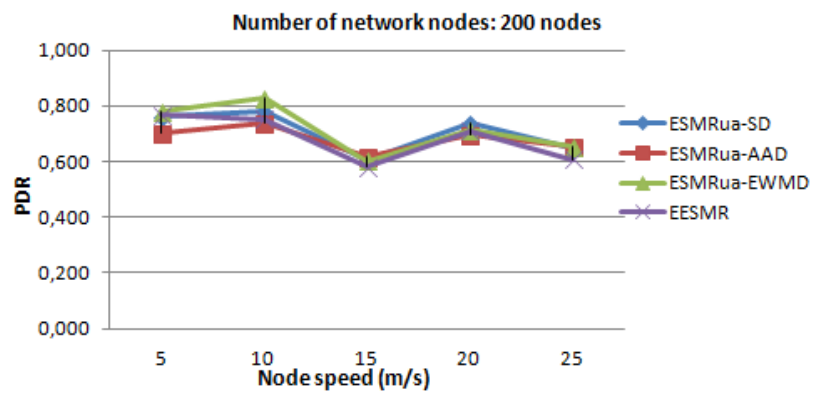

Fig. 7. PDR versus node speed (based on RWP model).

\section{CONCLUSION}

A new multipath routing protocol, ESMRua (Efficient energy-aware and link Stable Multipath Routing protocol in urban areas) has been proposed in this paper. It ensures the network reliability and reduces energy consumption in ad hoc mobile networks. The choice of path depends on a weighted function. Nodes energy and links stability are used to calculate this weight, in addition to the signal quality. We use three calculation variants to estimate the link stability based on the mobility of these nodes. For modeling, we choose two mobility models: the Random Waypoint model and a more realistic and non-random model the Manhattan Grid model, designed for urban areas. Through the performance evaluation, we noticed that our protocol improves the ad hoc mobile network performance compared to other routing protocols simulated in this paper and in our previous work. In future work, it will be interesting to analyze in depth the ESMRua protocol, focusing on the impact of weighting coefficients on the protocol performance.

\section{REFERENCES}

[1] J.N.Al-Karaki, G.A.Al-Mashaqbeh, S.Bataineh, "Routing protocols in wireless mesh networks: a survey," Int. Journal of Information and Communication Technology, vol. 11, pp. 445-495, 2017.

[2] Sherjung, RK.Sharma, "A Survey on Routing Protocols in Mobile Ad-hoc Networks," International Journal of Innovative Research in Computer and Communication Engineering, vol. 5, Issue 5, pp. 9813-9819 May 20172017.

[3] V.K.Quy, N.T.Ban, V.H.Nam, D.M.Tuan, N.D.Han, "Survey of Recent Routing Metrics and Protocols for Mobile Ad-Hoc Networks," Journal of Communications, vol. 14, no. 2, 2019.

[4] S.S.Deshpande, K.V.Asare, S.Deshpande, "An overview of Mobile Ad Hoc Networks for the Proactive, Reactive and Hybrid Routing Protocol," International Journal Of Engineering And Computer Science, vol. 2, pp. 1134-1143, 2013.

[5] S.E.Benatia, O.Smail, M.Boudjelal, B.Cousin, "ESMRsc: Energy Aware and Stable Multipath Routing Protocol for Ad Hoc Networks in Smart City," 2nd International Conference on Artificial Intelligence in Renewable Energetic Systems, ICAIRES 2018. Tipaza, Algeria, LNNS 62, pp. 31-42, 2019.

[6] N.S.Samshette, A.D.Bhoi, R.D.Kharadkar, "Analysis \& Comparison of Mobility Models for Ad-hoc Network," International Journal on Recent and Innovation Trends in Computing and Communication, vol. 2, no. 5, pp. 1357-1362, 2014.

[7] V.Arya, C.Gandhi, "Energy aware routing protocols for mobile ad hoc networks - a survey," International Journal of Information and Communication Technology, vol. 7, pp. 662-675, 2015.

[8] O.Smail, B.Cousin, R.Mekki, Z.Mekkakia, "A multipath energyconserving routing protocol for wireless ad hoc networks lifetime improvement," EURASIP Journal on Wireless Communications and Networking, vol. 139, pp. 1-12, 2014

[9] M.Bheemalingaiah, C.Venkataiah, K.Vinay Kumar, M.M.Naidu, D.Sreenivasa Rao, "Survey of Energy Aware On-demand Multipath Routing Protocols in Mobile Ad Hoc Networks," International Journal of Advanced Research in Computer Science and Software Engineering, vol. 6, pp. 212-222, 2016.

[10] A.Taha, R.Alsaqour, M.Uddin, M.Abdelhaq, T.Saba, "Energy Efficient Multipath Routing Protocol for Mobile Ad-Hoc Network Using the Fitness Function," IEEE Access, vol.5, pp.10369-10381, 2017.

[11] F.De Rango, F.Guerriero, P.Fazio, "Link-stability and energy aware routing protocol in distributed wireless networks," IEEE Transactions on Parallel and Distributed Systems, vol. 23, pp. 713 726, 2012.

[12] A.Moussaoui, A.Boukeream, "A survey of routing protocols based on link-stability in mobile ad hoc networks," Journal of Network and Computer Applications, vol. 47, pp. 1-10, 2015.

[13] A.Naushad, G.Abbas, Z.H.Abbas, L.Jiao, F.Muhammad, “A Novel Dynamic Link Connectivity Strategy Using Hello Messaging for Maintaining Link Stability in MANETs," Wireless Communications and Mobile Computing, vol. 2018, pp. 23, 2018.

[14] T.Roy, S.Roy, "Atypical Stable Multipath Routing Strategy in MANET," International Conference on Emerging Trends in Informatics and Communication (ICETIC), 2016.

[15] A.Yadav; A.S.Sairam; A.P.Mazumdar, "Selecting stable route in multipath routing protocols," 8th International Conference on Computing, Communication and Networking, Delhi, India, 2017.

[16] I.Alagiri, T.M.Selvan, P.Thanapal, C.Navaneethan, "Stable multipath routing protocol using different route selection mechanism for mobile Ad-hoc networks," International Journal of Pure and Applied Mathematics, vol. 117, no. 15, pp. 25-39, 2017.

[17] A.Pratapa Reddy, N.Satyanarayana, "Energy-efficient stable multipath routing in MANET," Wireless Networks, vol. 23, no. 7, pp. 2083-2091, 2017.

[18] V.Tilwari, K.Dimyati, M.N.Hindia, A.Fattouh, I.S.Amiri, "Mobility, Residual Energy, and Link Quality Aware Multipath Routing in MANETs with Q-learning Algorithm," Applied Sciences, vol. 9, no. 8, pp. 1-23, 2019.

[19] The Network Simulator ns-2. http://www.isi.edu/nsnam/ns.

[20] B.Tavli, W.Heinzelman, Mobile Ad Hoc Networks: Energy-Efficient Real-Time Data Communications. Springer Publisher; 2006 ; p. 265. 